reporting not testing or avoiding/delaying testing. Our study highlights the potential for an internet-based testing programme for $\mathrm{HIV}$ and STI under development in BC to reach men facing current barriers to HIV and STI testing.

\section{P3.426 THE NEED AND OPPORTUNITY TO PROMOTE COMBINATION PREVENTION IN HIV POSITIVE MEN WHO HAVE SEX WITH MEN ATTENDING CLINICS}

doi:10.1136/sextrans-2013-051184.0877

'S Wayal, ${ }^{2} \mathrm{~J}$ Cassell, ${ }^{1} \mathrm{~A}$ Copas, ${ }^{3} \mathrm{~S}$ Edwards, 'G Hart. 'University College London, London, UK; ${ }^{2}$ Brighton and Sussex Medical School, Falmer, UK; ${ }^{3}$ NHS Camden Provider Services, London, UK

Background In 2010 recent HIV diagnoses increased in the UK despite $80 \%$ of diagnosed HIV + MSM being on antiretroviral treatment (ART) with undetectable viral load. While undiagnosed HIV infections are thought to account for high proportion of these transmissions; a significant proportion is attributed to diagnosed HIV. We examine factors associated with unprotected anal intercourse with non-concordant partners (ncUAI) among HIV+ MSM and potential for HIV prevention.

Methods HIV+ MSM attending a central London HIV clinic completed a computer-assisted survey (May-September 2010). Multivariate logistic regression analyses were conducted.

Results Median age of 429 men was 43 years. 81\% of them were on ART. $17 \%$ of men had missed $\geq 1$ ART doses in the last two weeks. One in five men believed that being on ART with an undetectable viral load eliminates risk of HIV transmission. 380 men had been sexually active in the last year; $20 \%$ of whom had not tested for STI. $25 \%$ of those tested had been diagnosed with $\geq 1$ STI. $50 \%$ of men had a non-concordant primary partner. $50 \%$ of men's most recent sex partner was a non-primary non-concordant partner. The prevalence of ncUAI with primary and the most recent non-primary partner was $18 \%$ and 16.9 respectively. Duration of partnership, recreational drug use during sex, belief that undetectable viral load eliminates the risk of HIV transmission were all independently associated with ncUAI with a primary partner. Disclosure of HIV status and recreational drug use during sex were independently associated with ncUAI with the most recent non-primary partner. Viral load status was not associated with ncUAI.

Conclusions HIV + MSM attending HIV clinic reported high transmission risk behaviours. Engaging them in combination prevention interventions addressing recreational drug use, adherence to ART, attitudes towards ART for HIV prevention and STI testing during clinic visits provides vital opportunity for HIV/STI prevention.

\section{P3.427 EXPLORING THE BELIEFS, ATTITUDES, AND BEHAVIOURS OF MSM ENGAGED IN SUBSTANCE USE AND TRANSACTIONAL SEX IN GHANA}

doi:10.1136/sextrans-2013-051184.0878

'L Sabin, ${ }^{2} \mathrm{~T}$ Agyarko-Poku, ${ }^{3} Y$ A Abdul Rahman, ${ }^{3} \mathrm{~S}$ Wambugu, ${ }^{1} \mathrm{M}$ Bachman DeSilva, ${ }^{1} \mathrm{M}$ Esang, ${ }^{3} \mathrm{~K}$ Green, ${ }^{1} \mathrm{P}$ G Ashigbie, ${ }^{1} \mathbf{J}$ Beard, ${ }^{4} Y$ Adu-Sarkodie. ${ }^{1}$ Center for Global Health and Development, Boston University, Boston, MA, United States; ${ }^{2}$ Suntreso Hospital, Ghana Health Service, Kumasi, Ghana; ${ }^{3} F H I 360$ Ghana, Accra, Ghana; ${ }^{4 K w a m e}$ Nkrumah University of Science and Technology School of Medicine, Kumasi, Ghana

Background This qualitative study was designed to examine vulnerability to HIV infection and health-related needs among young men who have sex with men (MSM) in Kumasi, Ghana. Study objectives were to explore substance use (SU) and transactional sex (TS), overlap between SU and TS, beliefs related to SU and TS, and HIV knowledge and risk behaviours in this population.

Methods Adolescents (aged 15-17 years) and young adults (aged 18-29 years) who self-reported male-to-male sex in the previous 12 months and who either used alcohol or illicit substances or engaged in TS were recruited using snowball sampling. Data were collected through 44 in-depth interviews and eight focus group discussions, and analysed using OSR NVivo 10.0 software.

Results A total of 99 MSM participated. Most reported engaging in alcohol use and TS; a minority used substances, mainly marijuana. The majority displayed knowledge of HIV transmission and prevention, though few understood the basics of HIV treatment. Most perceived they were at high risk of HIV infection due to inconsistent condom use. Their risky behaviours also encompassed group sex, multiple sexual partners, and SU. Most expressed specific health needs, including education, screening, and treatment for sexually transmitted infections. They reported having only one clinic that provides services specific to MSM, and requested shorter wait times at facilities. Barriers to health and HIV services access included stigmatisation, ill-treatment by providers, poor quality of services, fear of testing positive, and lack of privacy, health insurance, and not knowing where to access services. Participants suggested legalising male-to-male sex, educating the public about MSM, and providing MSM with jobs to alleviate their challenges.

Conclusion Young MSM in Kumasi engage in high-risk behaviours and indicate a need for interventions to improve access to health and HIV-related services, increase HIV treatment knowledge, and reduce stigma among health providers and society.

\section{P3.428 FAMILIAL AND SOCIAL FACTORS INFLUENCING HIV/AIDS INTERVENTIONS IN RAWALPINDI, PAKISTAN}

doi:10.1136/sextrans-2013-051184.0879

\section{R H Tirmizi. NAZ Male Health Alliance, Lahore, Pakistan}

Background There are significant numbers of MSM in different parts of Islamic Republic of Pakistan including Rawalpindi. Religion and social norms do not promote/encourage acceptance of MSM at individual, familial and community level. MSMs generally face severe torture, neglect and deprivations associated to sexual identity. This study aims to provide insight to socio-familial factors that can affect HIV/AIDS related interventions by NMHA which is implementing GFATM's Regional Grant R-9.

Methodology Using snow-ball sampling technique and consent based inclusion the thirty participants were interviewed to get their case-study and FGD was conducted by 03 researchers included 02 self-identified MSMs from Rawalpindi and a clinical psychologist. NMHA's research-ethical standards were met with.

Results Almost every participant reported physical abuse by family members and deprivation from family property associated to disclosure of sexual identity. Undue psychological stresses caused due to family pressures to live in "rightly manner" and to "get married" which can lead to leaving home and living in sub-standard conditions resulting in health hazards. Educational and vocational activities are usually discontinued and most of the boys start living with friends with same sexual identity and engage in sex-work. Condom/ lubricant knowledge and practise in very low. Harassment and abuse and exploitation (physical/psychological/financial) by police and "network operators" is common. Condom use with wives is for contraception. Fear of being rejected/and from home is a barriers to disclosure to wives.

Conclusion Inclusion of family and PPTCT interventions along with socio-economic empowerment activities can be supportive in developing a rights based HIV prevention programme for MSMs in Rawalpindi. Further studies and a community lead programme and outreach strategy is highly recommended to support empowerment and building self-esteem. 